\title{
Product forms for stochastic Petri nets
}

\section{Richard J. Boucherie, CWI, Amsterdam}

In the literature on queueing networks in equilibrium product form distributions play a vital role, and many product form queueing networks have been discovered. For example Jackson networks, and their generalisation to BCMP-networks play a fundamental role in the queueing network literature. In contrast, for stochastic Petri nets product form results seem to be less common. An immediate explanation of the discrepancy between queueing networks and Petri nets is that resource sharing and competition over resources cannot be modelled by queueing networks. It are these properties of stochastic Petri nets that destroy product form equilibrium distributions. An interesting exception are the Petri nets described in Lazar and Robertazzi, where conditions are given under which a Petri net has a product form equilibrium distribution. These conditions basically come down to independence conditions on 'firing sequences' in subnets of the Petri net. As a consequence, also if competition over resources occurs a product form distribution is obtained. It must be noted here, that the results of this reference are valid for Petri nets containing linear firing sequences only, and that in each place of the Petri net at most one token is allowed. It is the purpose of this talk to show that the results of Lazar and Robertazzi can immediately be generalised to Petri nets consisting of arbitrary subnets if an 'independence condition' similar to the condition given in Lazar and Robertazzi is satisfied.

The framework used in this talk is that of a collection of Markov chains. For this collection the product process is introduced: let $S_{k}, q_{k}, k=1, \ldots, K$, be the state spaces resp. transition rates of the Markov chains in the collection, then $S=S_{1} \times \cdots \times S_{K}$ is the state space of the product process, and the transition rates of the product process in dimension $k$ are given by $q_{k}$. It is assumed in this framework that in each transition of the product process the state in one dimension changes only, that is in each transition of the product process one of the underlying Markov chains changes its state only. Competition over resources can then be modelled as exclusion of a part of the state space $S$, say the product process cannot enter $A \subset S$. The independence condition guaranteeing product form roughly states that if the product process is in state $\bar{n}=\left(\bar{n}_{1}, \ldots, \bar{n}_{k}, \ldots, \bar{n}_{K}\right)$, where $\bar{n}_{i} \in S_{i}$, and $\bar{n}^{\prime} \in A$, where $\bar{n}^{\prime}=\bar{n}$ except for component $\bar{n}_{k}\left(\right.$ i.e. $\left.\bar{n}_{k}^{\prime} \neq \bar{n}_{k}, \bar{n}_{i}=\bar{n}_{i}^{\prime}, i \neq k\right)$ then Markov chain $k$ cannot change its state. Under this condition the product process has a product form equilibrium distribution at $S \backslash A$ given by $\pi(\bar{n})=B \prod_{k=1}^{K} \pi_{k}\left(\bar{n}_{k}\right)$, where $\pi_{k}$ is the equilibrium distribution of Markov chain $k$ at state space $S_{k}$, and $B$ is a normalising constant determined by the exact form of $A$.

Lazar, A.A. and Robertazzi, T.G. (1991) Markovian Petri net protocols with product form solution, Performance Evaluation, 12, 67-77. 